\title{
Preparation and properties of a novel carbon nanotubes/poly(vinyl alcohol)/epidermal growth factor composite biological dressing
}

\author{
JUN-LIN LIAO ${ }^{1}$, SHI ZHONG ${ }^{1}$, SHAO-HUA WANG ${ }^{1}$, JIN-YAN LIU $^{1}$, JIA CHEN $^{1}$, GU HE $^{1}$, BIN HE $^{1,2}$, \\ JIA-QIN XU ${ }^{1,3}$, ZUN-HONG LIANG $^{1,3}$, TAO MEI ${ }^{1,4}$, SONG WU ${ }^{5}$, KE CAO $^{6}$ and JIAN-DAZHOU ${ }^{1}$ \\ ${ }^{1}$ Department of Plastic and Reconstructive Surgery, The Third Xiangya Hospital, Central South University, Changsha, \\ Hunan 410013; ${ }^{2}$ Department of Burn and Plastic Surgery, Ningxiang People's Hospital, Ningxiang, Hunan 410600; \\ ${ }^{3}$ Department of Burn and Plastic Surgery, Hainan General Hospital, Haikou, Hainan 570311; \\ ${ }^{4}$ Department of Burn and Plastic Surgery, The First People's Hospital of Changde, Changde, Hunan 415003; \\ Departments of ${ }^{5}$ Orthopaedics and ${ }^{6}$ Oncology, The Third Xiangya Hospital, Central South University,
} Changsha, Hunan 410013, P.R. China

Received April 7, 2016; Accepted March 6, 2017

DOI: $10.3892 /$ etm.2017.4752

\begin{abstract}
Wound dressings with drug delivery system have drawn increasing attention in skin damage recombination. Herein, a novel composite biological dressing was prepared and based on poly(vinyl alcohol) (PVA) combined with carbon nanotubes (CNTs) and epidermal growth factor (EGF) by electrospinning on gauze. The properties of the CNTs/PVA/EGF composite dressing were systemically investigated by general observation, and scanning electron microscopy (SEM). In vitro, the cytotoxicity of this dressing was investigated using a methyl thiazolyl tetrazolium (MTT) assay on L929 fibroblasts. In order to study the sustained release of EGF from this dressing, the concentration of EGF at different times was tested by ELISA. Furthermore, the biological activity of the released EGF was also evaluated using the MTT assay. Moreover, an in vivo experiment was conducted to observe whether this dressing was capable of improving healing in the model of wounded skin on rats. It was revealed that this dressing had a well-distributed microstructure by SEM. Additionally, the grade of cytotoxicity was low, and the EGF had a sustained release rate from this dressing. Furthermore, a maximum accumulative release rate of $12.47 \%$ was identified at $12 \mathrm{~h}$, and was retained at $9.4 \%$ after $48 \mathrm{~h}$. Simultaneously, the relative growth rate of L929 fibroblasts in the $12 \mathrm{~h}$ experimental group and $48 \mathrm{~h}$ group was 291.24 and $211.3 \%$,
\end{abstract}

Correspondence to: Professor Jian-Da Zhou, Department of Plastic and Reconstructive Surgery, The Third Xiangya Hospital, Central South University, 138 Tongzipu Road, Changsha, Hunan 410013, P.R. China

E-mail: doctorzhoujianda@163.com

Key words: biological dressing, poly(vinyl alcohol), carbon nanotubes, epidermal growth factor, nanofiber respectively. Next, the efficacy of these products was evaluated in vivo using Sprague-Dawley rats with a skin injury model. The healing of wounded skin of rats was sped up by this dressing based on the gross and histological appearances. From 7 to 10 days, the wounds in the experimental group were almost healed. In conclusion, this CNTs/PVA/EGF dressing had a well-distributed structure and an ability to release EGF at a sustained rate with the activity being favorable. On the basis of those results, a positive influence of designed dressing for accelerated wound healing was confirmed.

\section{Introduction}

Utilization of wound dressings is one of the most common methods used to clinically treat skin damage, and is significant in the daily clinical practice. The majority of traditional dressings have a lot of shortcomings, actually hinder wound healing and increase wound infection. Additionally, cellular granulation tissues can easily grow into gauze meshes (1). However, biological nanometer dressings have many advantages that can accelerate wound healing compared with traditional dressings, including biocompatibility, moisturizing efficacy, non-adhesion and antibacterial activation (2,3). Furthermore, biological dressings are mainly divided into natural materials, artificial synthetic materials and drug-loaded dressings. In addition, the drug dressing can play the role of drug loading, and further promote wound healing (4).

Wound healing is a process, which restores the physical integrity of body structures. It is a dynamic, complex, and multicellular process that involves the extracellular matrix (ECM), cytokines, blood cells, and growth factors. During this process, growth factors are proteins that activate and stimulate cell proliferation by activating angiogenesis, mitogenesis and gene transcription, thus accelerating healing (5). However, due to the enzymatic degradation or deactivation, or the fast diffusion and dryness of growth factors in the wound periphery, direct external employment of growth factors has 
not been found to display outstanding improvements in wound repair and healing $(6,7)$. Therefore, new potential therapeutic interventions in wound healing have focused on growth factors with sustained delivery, and growth factors contained within the wound dressing, and have been highlighted in the field of medical dressings (6-10).

Among numerous growth factors applied in the clinic, epidermal growth factor (EGF) is the first and foremost factor to promote the wound healing $(11,12)$. EGF possesses high-affinity receptors that are expressed by both fibroblasts and keratinocytes, and improves epidermal and mesenchymal regeneration, cell mobility, proliferation and synthesis of the ECM, then consequently facilitate wound healing $(13,14)$. To preserve EGF bioactivity, and also to protect the tissue in the reconstruction phase, an efficient approach could be embedded EGF into a suitable dressing, which provides the capability for sustained release of EGF when applied on top of the wound $(13,14)$.

Carbon nanotubes (CNTs) offer a spectrum of effective attributes due to their novel structure, non-toxicity, antibacterial and absorptive properties, as well as their extensive application to engineering scaffolds, drug release systems and nanosensors (15-18) since their discovery by Iijima in 1991 (19). Due to their excellent chemical and physical properties, several studies have suggested CNTs as a carrier designed to be a drug delivery system in cancer treatment $(20,21)$. However, there are no studies on CNTs as a drug delivery carrier material with recombinant human (rh)-EGF. In the present study, nanofibers of CNTs coated with rh-EGF were electrospun onto the surface of traditional gauze in order to stably release EGF. Stabilization of this CNTs/rh-EGF (hybrid) can be achieved by introducing them into the appropriate polymer matrix. Furthermore, poly(vinyl alcohol) (PVA) is a promising choice as a polymer matrix because of its biocompatibility and excellent features such as high water solubility, low cytotoxicity, easy acquisition and high transmittance (22).

The incorporation of a networked hybrid into the PVA matrix can significantly improve mechanical strength and water resistance (23). Moreover, PVA has been used in several biomedical applications such as developing medical materials (24-26). In the present study, PVA was used as a dispersant (27) and medium material and applied to electrospun non-woven fabrics on the gauze in order to close the meshes, so as to effectively prevent granulated tissue from growth inside. The main purpose of the present study was to fabricate and evaluate a novel CNTs/PVA/EGF composite biological nanometer dressing.

\section{Materials and methods}

Materials. CNTs (purity, >95\%) were acquired from Shenzhen Nanotech Port Co., Ltd. (Shenzhen, China). Concentrated sulphuric acid, concentrated nitric acid, sodium hydroxide and polyvinyl alcohol were all purchased from Hunan Xiangya Medicine (Changsha, China). Dulbecco's modified Eagle's medium (DMEM), fetal bovine serum, dimethyl sulfoxide, phosphate-buffered saline (PBS) and trypsin were all obtained from Gibco (Thermo Fisher Scientific, Inc., Waltham, MA, USA). The recombinant human EGF (rh-EGF) was obtained from Shenzhen Watsin Genetech Ltd. (Shenzhen, China). The enzyme-linked immunosorbent assay (ELISA) kit for EGF was purchased from QNAP systems, Inc. (New Taipei, Taiwan). Methyl thiazolyl tetrazolium (MTT) reagent was obtained from Sigma-Aldrich (Merck KGaA, Darmstadt, Germany) and the L929 mouse fibroblast cell-line was provided by Nanjing KeyGen Biotech Co., Ltd. (Nanjing, China).

\section{Preparation of CNTS/PVA/EGF composite dressing}

Purification and activation of CNTs. A total of $0.15 \mathrm{~g}$ CNTs were dissolved in $150 \mathrm{ml}$ of concentrated sulfuric and nitric acid at a ratio of 3:1 in a flat bottom flask, with ultrasonic dispersion at $99 \mathrm{~W}$ for $30 \mathrm{~min}$ and at room temperature. Furthermore, acidification was performed for $1 \mathrm{~h}$ in a water bath at $100^{\circ} \mathrm{C}$. Next, the solution of acid-treated CNTs was filtered by suction filtration and washed in distilled water to a neutral $\mathrm{pH}$, and then dried at $70^{\circ} \mathrm{C}$. The dried CNTs were then dissolved in $50 \mathrm{ml}$ of distilled water with ultrasonic dispersion at $99 \mathrm{~W}$ for $30 \mathrm{~min}$ and at room temperature. Additionally, insoluble CNTs were removed by centrifugation at 7,104 $\mathrm{x} g$ at room temperature for $20 \mathrm{~min}$. Next, the solution of acid-treated CNTs were dried by infrared (IR) analysis (FT-NIR ANALYZER, WQF-410; ABB Group, Zürich, Switzerland).

Dispersion and modification with PVA. Next, $0.2 \mathrm{~g}$ of acid-treated CNTs were placed in $18.1 \mathrm{ml}$ deionized water, and the solution was stirred for up to $1 \mathrm{~h}$ with ultrasonic dispersion for up to $1 \mathrm{~h}$. Subsequently, $1.7 \mathrm{~g}$ PVA was dissolved in the liquid and heated to $100^{\circ} \mathrm{C}$ in a water bath for $2 \mathrm{~h}$. The sample was homogeneous and stable, and no precipitation or dispersion of flocculation was observed. Furthermore, some of the product was kept to dissolve in anhydrous ethanol, and then filtered. When the temperature of the solution cooled down to $30^{\circ} \mathrm{C}, 20 \mu \mathrm{g}$ rh-EGF was added in the mixed fluid at a temperature of $30^{\circ} \mathrm{C}$ in a water bath for $30 \mathrm{~min}$. The synthesis process of CNTs/PVA/EGF composite is shown in Fig. 1.

Electrostatic spinning. In the present study, the solution for electrostatic spinning was acquired, which included $8.5 \% \mathrm{w} / \mathrm{w}$ PVA, $1 \% \mathrm{w} / \mathrm{w}$ CNTs and $10 \mu \mathrm{g} / \mathrm{ml}$ growth factor. Eventually the solution was electrospun to nanofibers alone or on the surface of gauze or tinfoil under a voltage of $20-50 \mathrm{kV}$, with an injection angle of $90^{\circ}$. Furthermore, the nozzle had a $0.5 \mathrm{~mm}$ diameter and a rate of $0.5 \mathrm{ml} / \mathrm{h}$.

Sterilization. The CNTs/PVA/EGF nanodressings were kept at $4^{\circ} \mathrm{C}$ or lower after sterilization by alcohol at a concentration of $70 \%$.

Dressing characterization. A scanning electron microscope (SEM; JSM5600-LV, JEOL, Ltd., Japan) with an accelerating voltage of $15 \mathrm{kV}$ was employed to observe the morphology of the fibers. Prior to the observation, the specimens were sputter-coated with gold. Furthermore, Image-J analysis software (National Institute of Health, Bethesda, MD, USA) was used to measure the average diameter of the fibers.

Preparation of cells. The L929 mouse fibroblast cell-line was provided by Nanjing KeyGen Biotech Co., Ltd. The cells were cultured in DMEM supplemented with $10 \%$ fetal bovine serum (FBS; Thermo Fisher Scientific, Inc.), $100 \mathrm{U} / \mathrm{ml}$ penicillin 


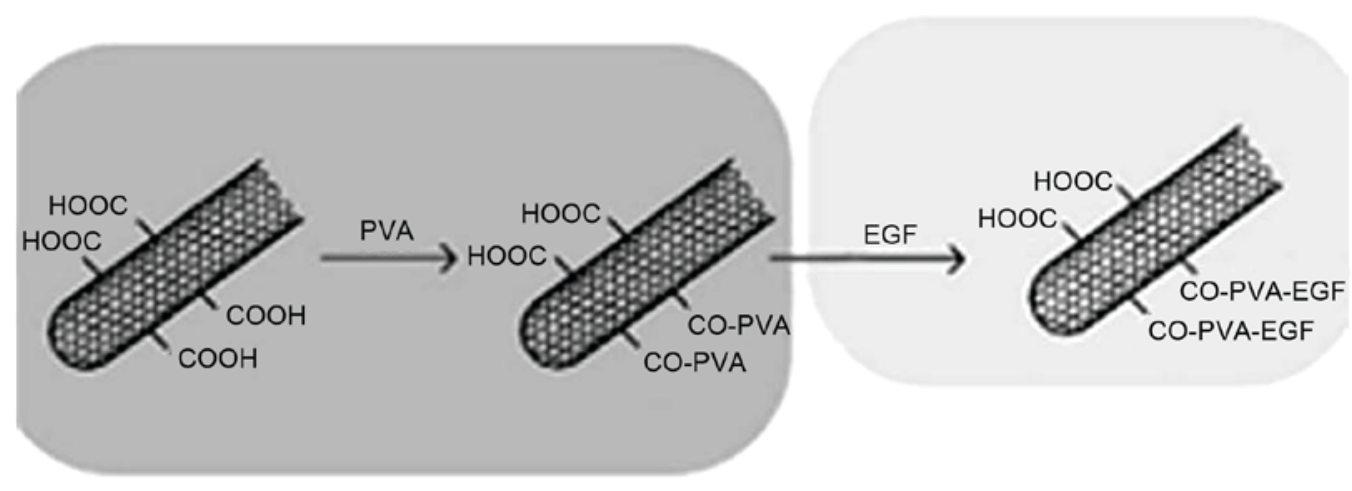

Figure 1. Schematic diagram of the synthesis process of carbon nanotube/poly(vinyl alcohol)/epidermal growth factor composite.

and $100 \mu \mathrm{g} / \mathrm{ml}$ streptomycin in a humidified atmosphere of $5 \% \mathrm{CO}_{2}$ in air at $37^{\circ} \mathrm{C}$. After reaching confluence, the cells were harvested and suspended in DMEM, and the concentration of cells was adjusted to $1 \times 10^{4}$ cells $/ \mathrm{ml}$.

Preparation of dressing leaching solution. The CNTs/ $\mathrm{PVA} / \mathrm{rh}-\mathrm{EGF}$ nanodressings $(1 \mathrm{x} 1 \mathrm{~cm})$ were placed in $10 \% \mathrm{FBS}$, with leaching at $37^{\circ} \mathrm{C}$ for $24 \mathrm{~h}$. Next, the dressing leaching solutions defined as experimental group $1(n=6)$ were obtained with a concentration of $100 \%$, and experimental group $2(n=6)$ was obtained with a concentration of $50 \%$. Additionally, the PVA electrostatic spinning dressings $(1 \times 1 \mathrm{~cm})$ were placed in $10 \% \mathrm{FBS}$, with leaching at $37^{\circ} \mathrm{C}$ for $24 \mathrm{~h}$ as the control group $(n=6)$.

Cytotoxicity in vitro. In order to assess the cell viability, an MTT colorimetric assay was applied. The cultured cells were treated with the above three groups of the dressing leaching solutions respectively. The samples were read by a microplate reader at 24 and $48 \mathrm{~h}$ after the reaction with the MTT reagent. Additionally, a microplate reader set at a wavelength of $450 \mathrm{~nm}$ was employed for testing OD values, and the relative growth rate (RGR) of cells (\%) was calculated using the following formula:

$$
\operatorname{RGR}(\%)=\frac{\text { Experimental }(O D)}{\text { Control }(O D)} \times 100 \%
$$

EGF release and activation. The $\mathrm{CNTs} / \mathrm{PVA} / \mathrm{EGF}$ nanodressings $(5 \times 5 \mathrm{~cm})$ were leached into PBS containing $1 \%$ penicillin/streptomycin and $1 \%$ amphotericin as antimicrobial biocides. Next, the accumulative release rates (ARRs) of EGF in the solutions were tested by ELISA on the basis of the manufacturer's instructions at 5, 15 and $30 \mathrm{~min}, 1,3,9,12$, 24 and $48 \mathrm{~h}$. Furthermore, the activation of EGF was evaluated by the RGR of cells in the same way as that described above at 5,15 and $30 \mathrm{~min}, 1,3,9,12,24$ and $48 \mathrm{~h}$.

Wound healing experiments with the CNTS/PVA/EGF dressing. A total of 12 Sprague-Dawley (SD) rats (4 months old; 200-250 g; male: female, 1:1) were used in the wound healing assay. Rats were housed at $24^{\circ} \mathrm{C}$ in $n$ environmetn with $50 \%$ humidity and a 16/8 h light/dark cycle with free access to food and water. Rats were anesthetized with $4 \%$ chloral hydrate $(350 \mathrm{mg} / \mathrm{kg}$; Qingdao Yulong Algaue, Ltd.,
Qingdao, China), and a skin wound model with a diameter of $1 \mathrm{~cm}$ was set up on their back. The wounds were randomly assigned to the CNTs/PVA/EGF experimental and normal saline control groups $(n=6)$. Additionally, the dressings on their back were fixed and closed with silica gel thin film sutured on the wounds, and were removed when images were captured. Images of the wounds were captured daily and at the time of wound healing. Furthermore, the non-healing area was calculated using a computer image analysis system (CAD software version 18.0.55.0; Autodesk, Ltd., San Rafael, CA, USA). The healing rate was calculated as follows: Healing rate $=$ (Initial wound area-non-healing area)/initial wound area $\mathrm{x} 100$.

The SD rats were provided by the Animal Experiment Center of the Third Xiangya Hospital of Central South University. The Ethics Committee of the Third Xiangya Hospital approved the study, and all procedures complied with the HuNan Guide for the Care and Use of Laboratory Animals.

Statistical analysis. Data are expressed as the mean \pm standard deviation. Furthermore, the significance among groups or intragroup differences was also assessed. The statistical significance of treatments was determined by two-tailed, Student's t-test at a $95 \%$ confidence interval $(\mathrm{P}<0.05)$.

\section{Results}

Analysis of IR spectra of CNTs before and after purification. The IR of CNTs before (bottom curve) and after (top curve) purification by acidification are presented in Fig. 2. It can be observed that the CNTs had no absorption peak in the vicinity of $1,690 / \mathrm{cm}$ in the bottom curve, which indicated that the surface of CNTs did not have carboxyl. However, after treatment with acidification, the CNTs had an evident carboxyl $\mathrm{C}=\mathrm{O}$ absorption peak at $1,699.8 / \mathrm{cm}$, which showed that the mixed sulfuric and nitric acid can induce CNTs activity.

General observations. The images of the sample of the wound dressing are shown in Fig. 3. The non-woven fabrics of CNTs/PVA/EGF were formed to a black thin film by electrostatic-spinning, and their edges tended to easily curl and fracture after strenuous retraction (Fig. 3A). After electrostatic-spinning the non-woven fabric onto the gauze, the color of this compound on the dressings was light black as a whole. 


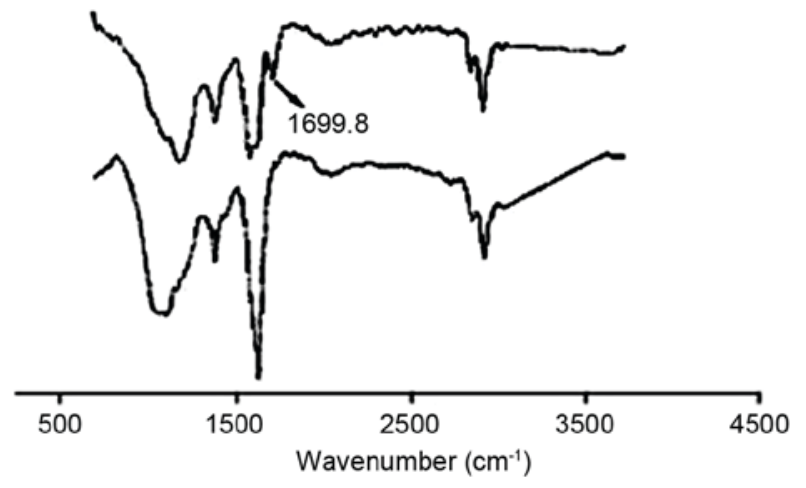

Figure 2. Analysis of infrared spectra of carbon nanotubes before (below) and after (above) purification.

By contrast, the non-woven fabric of CNTs/PVA/EGF was a uniform distribution that was decreased on the surfaces of the gauze, but was more resilient than traditional gauze (Fig. 3B).

SEM observations. The SEM images of the CNTs/PVA/EGF composite surface of the wound dressings are shown in Fig. 4. The non-woven fabrics of CNTs/PVA/EGF was electrostatically spun on the surface of the gauze (Fig. 4A and B) (magnification, $\mathrm{x} 30$ and $\mathrm{x} 500$ ) and tin foils (Fig. 4C and D) (magnification, $x 1,000$ and x 5,000), respectively. An evident fiber structure could easily be observed. Furthermore, there were many non-woven fabrics that covered the surface, filled the holes of these gauze fabrics and touched each other (Fig. 4A and B). Simultaneously, the non-woven fabrics were crisscrossed and contorted into straight lines on the surface of the tin foils. Additionally, the fractured non-woven fabrics were not observed at high power (Fig. 4C and D), and the size was $\sim 500 \mathrm{~nm}$ (Fig. 4D).

In vitro cytotoxicity and biocompatibility. The L929 mouse fibroblasts were well grown in both the control and experimental groups after 24 and $48 \mathrm{~h}$ in vitro (Fig. 5). The correlation between the RGR and cytotoxical grade (CG) are shown in Table I. In addition, the RGR of cells tested by the MTT assay were $117.6 \%$ in the experimental group 1 and $112.5 \%$ in the experimental group 2 after $24 \mathrm{~h}$, then 137.3 and $125.7 \%$ after $48 \mathrm{~h}$ respectively (Tables II and III). On the basis of a correlation between the RGR and the CG in the MTT assay, the grade of cytotoxicity was at the zero level.

EGF stability and release in cell culture medium. Evaluation of in vitro stability and the release of loaded EGF in cell culture medium are important for predicting the potential of developed CNTs/PVA/EGF composite for therapeutic applications. To date, there are not many reports available in the literature about describing the in vitro release profile and stability of EGF loaded in CNTs/PVA nanofiber by an electrostatic-spinning method. The present study evaluated the release profile of EGF loaded in CNTs/PVA composite using ELISA. The results indicate that the ARRs of EGF increased steadily, but not rapidly before $12 \mathrm{~h}$ as shown in Table IV. Moreover, the highest ARR of $12.47 \%$ with EGF was observed at the $12 \mathrm{~h}$ time point after the experiment began, and remained at $9.4 \%$ after $48 \mathrm{~h}$. Furthermore, the reduction in the quantity of EGF

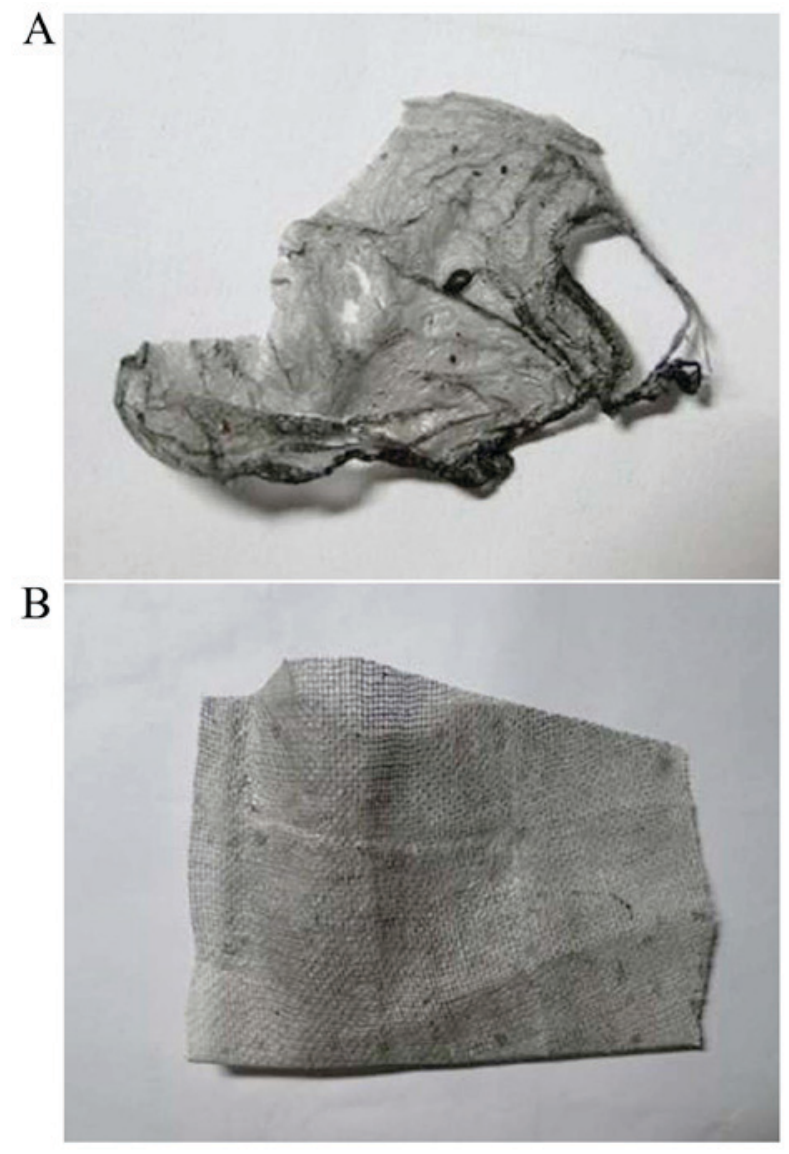

Figure 3. General observation (A) non-woven fabric; (B) non-woven fabric on gauze.

observed in the released fraction at later time points may be partially associated with the stability of released EGF in the medium. These results clearly indicate that EGF loaded in CNTs/PVA composite is stable in a normal growth medium for extended period of times.

EGF activation. In the present study, activation of EGF was indicated indirectly by the RGR of L929 cells after being cultured in the dressing leaching solution and normal saline in a time-dependent manner. It was revealed that the cultured cells were maintained in good condition after $48 \mathrm{~h}$ as shown in Fig. 6. Additionally, the RGR of cells constantly and steadily increased with the passage of time up to $291.24 \%$ at $12 \mathrm{~h}$, and remained at $211.3 \%$ at $48 \mathrm{~h}$, which was statistically significantly different compared with the control (Table V).

Wound healing in skin injury model rats. Wound healing in skin injury model rats was shown in Fig. 7, and all wounds were not infected with good wound care. The wound-healing rate at 3 days post-treatment in each group ranged between $15.225 \pm 1.034$ and $16.726 \pm 1.634 \%$ with no difference observed between the groups $(\mathrm{P}>0.05)$. At 7 and 10 days, the healing rate in the experimental group was $60.19 \pm 3.837$ and $95.033 \pm 6.247 \%$, respectively, which was significantly higher than those in the control group $(\mathrm{P}<0.05)$. Furthermore, the wound-healing duration in the experimental group was $11.65 \pm 1.503$ days, which was significantly shorter than that of the control group $(14.533 \pm 1.033$ days; $\mathrm{P}<0.05)$. Thus, these 

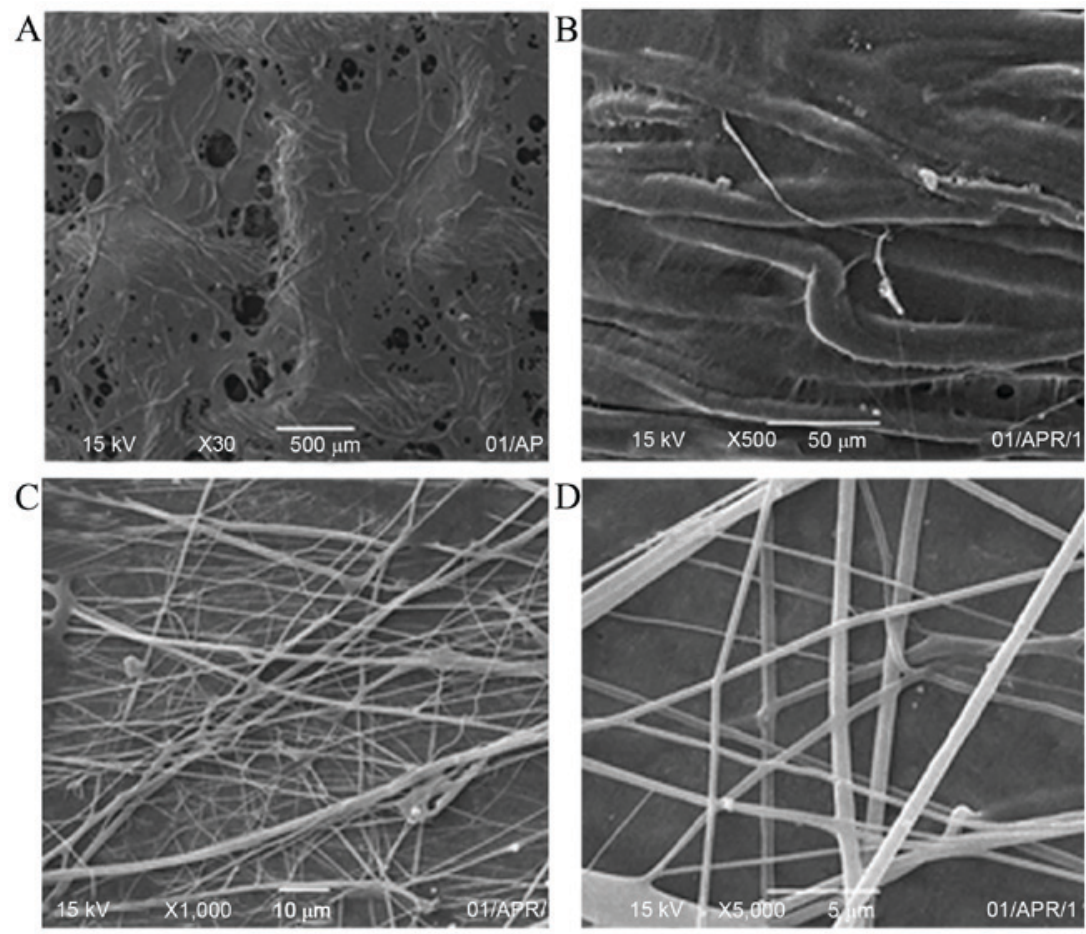

Figure 4. Scanning electron microscopy images of nanofibers of carbon nanotubes/poly(vinyl alcohol)/epidermal growth factor (A and B) on gauze; (C and D) on tinfoil.
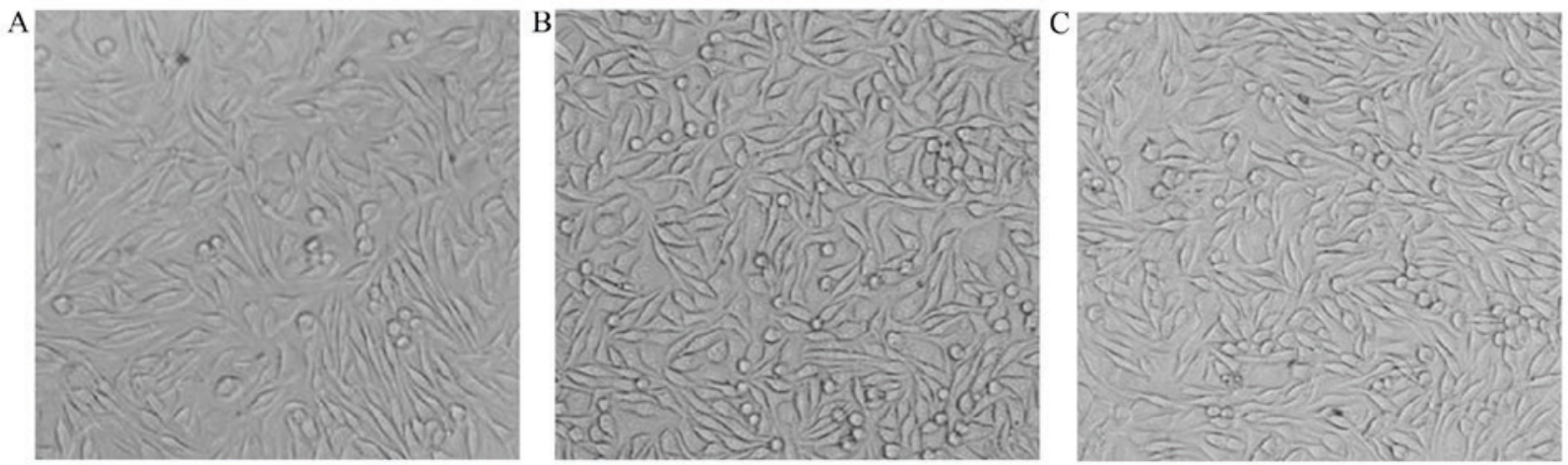

Figure 5. Morphocytology of L929 mice fibroblasts after 48 h (magnification, x100). (A) Control, (B) experimental group 1 and (C) experimental group 2.

results demonstrated that the wound-healing rate and duration in the experimental group were higher and shorter than that in the control group.

\section{Discussion}

The present study demonstrates a novel biological composite dressing for treating skin wounds, which can effectively release EGF by keeping an acceptable bioactivity at a sustained rate. In this dressing, CNTs were important as a carrier material coated with EGF. Meanwhile, PVA was used as a modifier and dispersant for CNTs, which can generate a strong binding force with substrate material for its small grain size and large specific surface area (28). The possible synthesis process of the CNTs/PVA/EGF composite in this system is shown in Fig. 1.

In order to activate CNTs, hydroxyl and carboxyl were initially introduced to the surface by dissolving in concentrated sulfuric and nitric acid. Additionally, the activation of CNTs was verified by IR analysis as shown in Fig. 2. Next, CNTs were dispersed by PVA, which could accelerate to form a uniform suspension and keep the CNTs original features (27).

Electrospun meshes provide a high-surface area, microporosity, and the ability to load drugs or other biomolecules onto the fibers (29). In the present study, nanofibers of CNTs/PVA/EGF were successfully fabricated by electrospinning on the double-side of traditional gauze just like a superposition shape of three layers as shown in Fig. 3, and subsequently the morphology and characteristics of the nanofibers were observed. It was revealed that this novel dressing is more resilient than traditional gauze. In a low power SEM observation, the traditional gauze meshes were mostly closed by these nanofibers with different levels, which could effectively prevent granulated tissue growth. In addition, through high power SEM observation, the gaps between these nanofibers were clearly shown to ensure air circulation from the wound. 
Table I. Association between the RGR and cytotoxical grade.

\begin{tabular}{|c|c|c|c|}
\hline \multicolumn{3}{|l|}{ RGR (\%) } & CG \\
\hline \multicolumn{3}{|l|}{$\geq 100$} & 0 \\
\hline \multicolumn{3}{|l|}{$75-99$} & 1 \\
\hline \multicolumn{3}{|l|}{$50-74$} & 2 \\
\hline \multicolumn{3}{|l|}{$25-49$} & 3 \\
\hline \multicolumn{3}{|l|}{$0-24$} & 4 \\
\hline \multicolumn{3}{|l|}{0} & 5 \\
\hline \multicolumn{4}{|c|}{ RGR, relative growth rate; $C G$, cytotoxical grade. } \\
\hline \multicolumn{4}{|c|}{ Table II. Results of OD value, RGR and CG at 24 h $(n=6)$. } \\
\hline Variable & $\begin{array}{l}\text { Control } \\
\text { group }\end{array}$ & $\begin{array}{l}\text { Experimental } \\
\text { group } 1\end{array}$ & $\begin{array}{c}\text { Experimental } \\
\text { group } 2\end{array}$ \\
\hline OD & $0.664 \pm 0.056$ & $0.781 \pm 0.039$ & $0.747 \pm 0.050$ \\
\hline RGR (\%) & 100 & 117.60 & 112.50 \\
\hline $\mathrm{CG}$ & 0 & 0 & 0 \\
\hline
\end{tabular}

OD, optical density; RGR, relative growth rate; CG, cytotoxical grade.

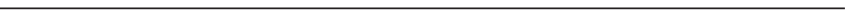

Table III. Results of OD value, RGR and CG at $48 \mathrm{~h}$.

\begin{tabular}{lccc}
\hline Variable & $\begin{array}{c}\text { Control } \\
\text { group }\end{array}$ & $\begin{array}{c}\text { Experimental } \\
\text { group 1 }\end{array}$ & $\begin{array}{c}\text { Experimental } \\
\text { group 2 }\end{array}$ \\
\hline OD & $1.180 \pm 0.152$ & $1.620 \pm 0.251$ & $1.484 \pm 0.270$ \\
RGR $(\%)$ & 100 & 137.30 & 125.70 \\
CG & 0 & 0 & 0 \\
\hline
\end{tabular}

OD, optical density; RGR, relative growth rate; CG, cytotoxical grade.

One of the experiments in the present study adopted the indirect contact method to assess the cytotoxicity of this dressing by routinely detecting the toxicity of lixivium using the MTT assay. The results indicated that the L929 fibroblasts were viable in both the control and experimental groups after 24 and $48 \mathrm{~h}$ in vitro. In addition, the RGRs of L929 fibroblasts were $117.6 \%$ in the experimental group 1 and $112.5 \%$ in the experimental group 2 after $24 \mathrm{~h}$, and then went up to 137.3 and $125.7 \%$, respectively, after $48 \mathrm{~h}$. It turned out that this dressing was not cytotoxic to L929 fibroblasts and had the characteristics of being biocompatible, which could meet the basic requirements of biological dressings (30). On the basis of the association between the RGR and CG, it indicated that the dressings were safe to L929 fibroblasts.

Due to the instability of EGF as direct topical employment on skin wounds, numerous studies focused on introducing a sustained delivery system to overcome this disadvantage. For example, it has been reported that EGF was conjugated
Table IV. The results of OD value and ARR $(n=6)$.

\begin{tabular}{llr}
\hline Time & \multicolumn{1}{c}{ OD } & ARR $(\%)$ \\
\hline $5 \mathrm{~min}$ & $0.394 \pm 0.021$ & 3.18 \\
$15 \mathrm{~min}$ & $0.545 \pm 0.018$ & 4.41 \\
$30 \mathrm{~min}$ & $0.738 \pm 0.036$ & 5.96 \\
$1 \mathrm{~h}$ & $0.917 \pm 0.021$ & 7.41 \\
$3 \mathrm{~h}$ & $1.143 \pm 0.05$ & 9.24 \\
$9 \mathrm{~h}$ & $1.330 \pm 0.012$ & 10.75 \\
$12 \mathrm{~h}$ & $1.543 \pm 0.096$ & 12.47 \\
$24 \mathrm{~h}$ & $1.367 \pm 0.041$ & 11.05 \\
$48 \mathrm{~h}$ & $1.163 \pm 0.083$ & 9.40 \\
\hline
\end{tabular}

OD, optical density; ARR, accumulative release rate.

Table V. Results of OD value and RGR.

\begin{tabular}{lcc}
\hline Time & OD & RGR $(\%)$ \\
\hline Normal saline & $0.310 \pm 0.012$ & 100.00 \\
$5 \mathrm{~min}$ & $0.349 \pm 0.019$ & 112.70 \\
$15 \mathrm{~min}$ & $0.447 \pm 0.003$ & 144.24 \\
$30 \mathrm{~min}$ & $0.477 \pm 0.014$ & 153.93 \\
$1 \mathrm{~h}$ & $0.533 \pm 0.007$ & 172.01 \\
$3 \mathrm{~h}$ & $0.606 \pm 0.013$ & 195.80 \\
$9 \mathrm{~h}$ & $0.719 \pm 0.012$ & 232.19 \\
$12 \mathrm{~h}$ & $0.901 \pm 0.034$ & 291.24 \\
$24 \mathrm{~h}$ & $0.724 \pm 0.017$ & 233.69 \\
$48 \mathrm{~h}$ & $0.654 \pm 0.012$ & 211.30 \\
\hline
\end{tabular}

OD, optical density; RGR, relative growth rate.

on the surface of electrospun polycaprolactone nanofibers that were functionally synthesized with amine groups. These manufactured EGF nanofibers markedly improved the in vivo wound healing process in comparison to direct application of EGF-containing solutions (31). Furthermore, this CNTs/PVA/EGF composite dressing can also play a role in stably releasing growth factors, according to the results of the EGF release assays, where the ARR of EGF increased steadily but not rapidly before $12 \mathrm{~h}$. Moreover, a maximum ARR of $12.47 \%$ was found at $12 \mathrm{~h}$, and was sustained at $9.4 \%$ after $48 \mathrm{~h}$. It was confirmed that the release of growth factors from this dressing reached a peak at the point of $12 \mathrm{~h}$, then declined after $48 \mathrm{~h}$ as a result of deactivation of the early released growth factors that have a short half-life. In addition, the viability of EGF release was reflected indirectly by the RGR of L929 fibroblasts after being cultured in this dressing leaching solution and normal saline at different time points. It turns out that the cultured cells were maintained in good shape after $48 \mathrm{~h}$. The RGR of cells constantly and steadily increased with the passage of time, and reached $291.24 \%$ at $12 \mathrm{~h}$. Furthermore, it was sustained at $211.3 \%$ at $48 \mathrm{~h}$. Additionally, it was proven that the viability of EGF released was maintained so well that it accelerated the growth of the L929 fibroblasts. 

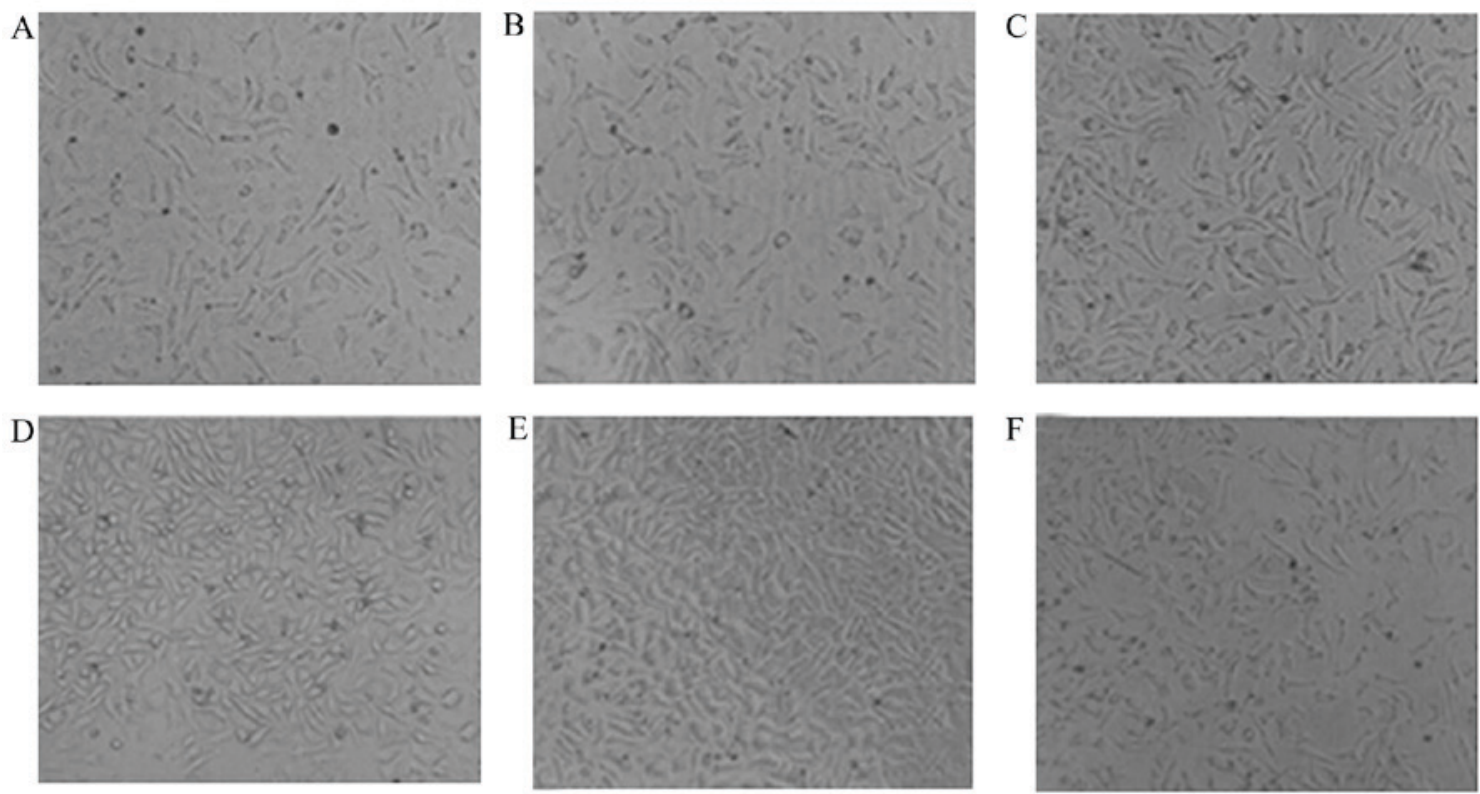

Figure 6. Morphology of L929 mice fibroblasts after cultured in the dressing leaching solution and normal saline for a period of time, and continued to culture for $48 \mathrm{~h}$. (magnification, x40). (A) Cultured in normal saline; (B) cultured in dressing leaching solution for $15 \mathrm{~min}$; (C) $1 \mathrm{~h}$; (D) $9 \mathrm{~h}$; (E) $12 \mathrm{~h} \mathrm{and} \mathrm{(F)} 48 \mathrm{~h}$.
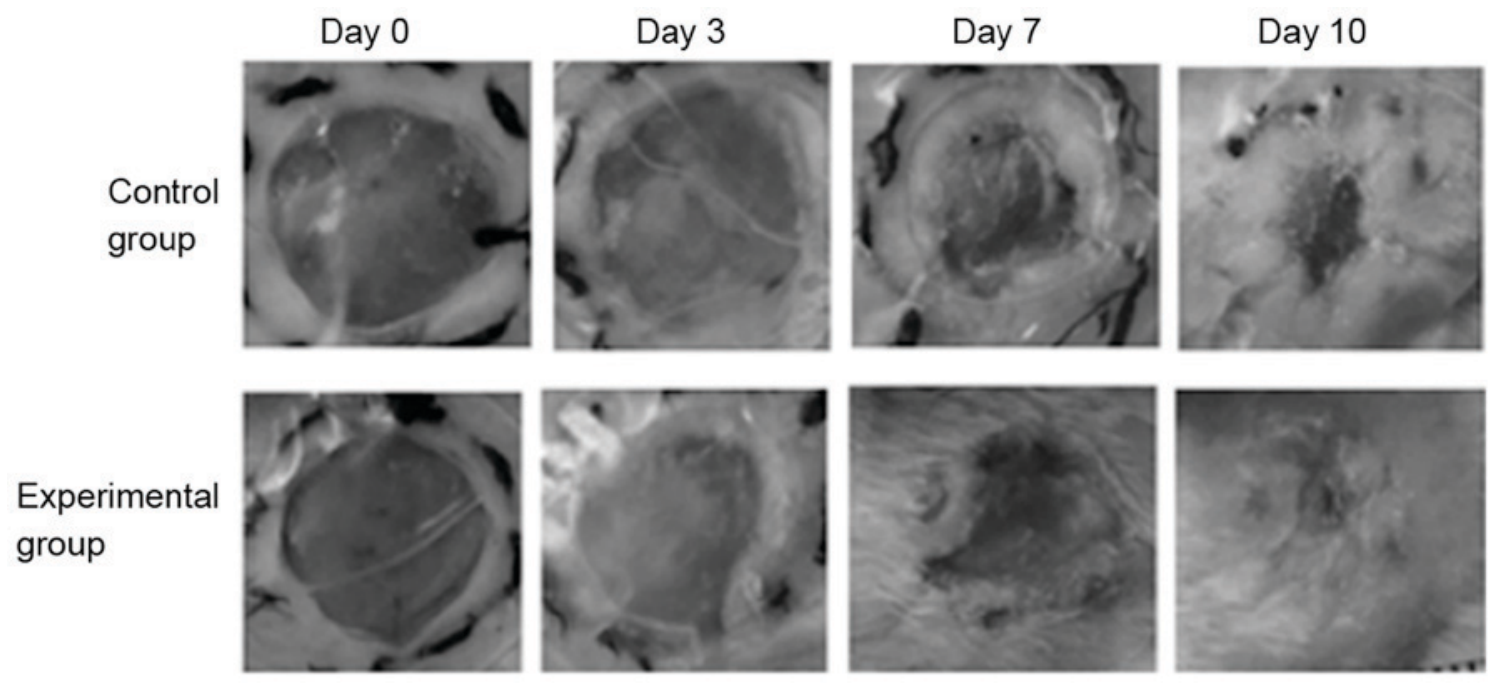

Figure 7. Wound contract at different time points. On day 1 and 2 after wound creation, the wound contraction had no difference between the two groups. After day 3 , the wound contraction of the experimental group of rats handled with this dressing gradually became faster, as compared with control group. And from day 7 to day 10, the wounds of rats in the experimental group were almost healed. Magnification, $\mathrm{x} 20$.

Experiments were also performed on rats with a skin injury model. The wound conditions of treatment with this dressing evaluated based on the gross and histological appearances still verified that it could improve skin wound healing. Beginning from 7 days, the wound treated with this dressing showed a faster recovery rate than the control group. This result strongly suggested that EGF was liberated from CNTs/PVA/EGF nanofibers in response to degradation of PVA in the skin wound. This post-release process subsequently proceeded to assist wound-healing process (32). Furthermore, it was reported that a wound dressing composed of hyaluronic acid and collagen sponge containing EGF could promote a decrease in wound size, in accordance with re-epithelialization and granulation tissue formation associated with angiogenesis (33).
Although a novel CNTs/PVA/EGF composite biological dressing has successfully been created and its properties investigated, there are still many limitations in the present study. For example, this novel dressing was not compared with others used in the clinic, which would otherwise have permitted us to emphasize its advantages. Furthermore, no bacteriostatic tests were performed. Finally, whether this novel dressing promoted fibroblasts to release other cytokines was not investigated. Therefore, the goal of our future studies will be to investigate these issues discussed, which will also involved modeling the utility of this dressing.

In conclusion, the electrospun CNTs/PVA/EGF composite biological dressing had a well-distributed structure and displayed an improved ability to release EGF at a sustained rate with the activity of the EGF released being favorable. The 
bioactively released EGF also accelerated the growth of L929 fibroblasts, which showed its potential application in wound dressing. Additionally, an in vivo experiment showed that the healing of wounded skin of rats was sped up by this dressing. On the basis of the results of the in vitro and in vivo assays, the positive influence of designed dressings for accelerated healing of a wound model was confirmed.

\section{Acknowledgements}

The present study was supported by the Independent Exploration and Innovation Project of Central South University (grant no. 2016ZZTS155).

\section{References}

1. Zhau L and Song JX: Progress in wound dressings. J Clin Reha Tis Eng Res 11: 1724-1726, 1737, 2007 (In Chinese).

2. Rošic R, Kocbek P, Pelipenko J, Kristl J and Baumgartner S: Nanofibers and their biomedical use. Acta Pharm 63: 295-304, 2013.

3. Hartstein B, Gausche-Hill M and Cancio LC: Burn injuries in children and the use of biological dressings. Pediatr Emerg Care 29: 939-948, 2013.

4. Zhang J, Hao J, Zhang J, Luo B and Liu P: Development research of biological dressing. Zhongguo Xiu Fu Chong Jian Wai Ke Za Zhi 29: 254-259, 2015 (In Chinese)

5. João De Masi EC, Campos AC, João De Masi FD, Ratti MA, Ike IS and João De Masi RD: The influence of growth factors on skin wound healing in rats. Braz J Otorhinolaryngol 82: 512-521, 2016.

6. Yang Y, Xia T, Zhi W, Wei L, Weng J, Zhang C and Li X: Promotion of skin regeneration in diabetic rats by electrospun core-sheath fibers loaded with basic fibroblast growth factor. Biomaterials 32: 4243-4254, 2011.

7. Biondi M, Ungaro F, Quaglia F and Netti PA: Controlled drug delivery in tissue engineering. Adv Drug Deliv Rev 60: 229-242, 2008.

8. Boateng JS, Matthews KH, Stevens HN and Eccleston GM: Wound healing dressings and drug delivery systems: A review. J Pharm Sci 97: 2892-2923, 2008.

9. Wen X and An SJ: Progress of growth factors containing wound dressing. Chem Ind Eng Prog 8: 1435-1440, 2009 (In Chinese).

10. Zhou JD, Wang SH, Liu R, Zhou CJ, Cao K, Liu JY, Chen Y and Chen FH: Study of the biological effectiveness of a nanosilver-epidermal growth factor sustained-release carrier. Exp Ther Med 5: 1231-1235, 2013.

11. Esquirol Caussa J and Herrero Vila E: Epidermal growth factor, innovation and safety. Med Clin (Barc) 145: 305-312, 2015 (In Spanish).

12. Deng CL, Yang GH and Cao YL: The progress research of growth factor promoting skin wound healing. Zuzhi Gongcheng Yu Chongjian Waike Zachi 2: 174-176, 2006 (In Chinese).

13. Choi JK, Jang JH, Jang WH, Kim J, Bae IH, Bae J, Park YH, Kim BJ, Lim KM and Park JW: The effect of epidermal growth factor (EGF) conjugated with low-molecular-weight protamine (LMWP) on wound healing of the skin. Biomaterials 33: 8579-8590, 2012.

14. Schneider A, Wang X, Kaplan D, Garlick J and Egles C: Biofunctionalized electrospun silk mats as a topical bioactive dressing for accelerated wound healing. Acta Biomater 5: 2570-2578, 2009.
15. Schiffman JD and Elimelech M: Antibacterial activity of electrospun polymer mats with incorporated narrow diameter single-walled carbon nanotubes. ACS Appl Mater Interfaces 3: 462-468, 2011.

16. Liu S, Ng AK, Xu R, Wei J, Tan CM, Yang Y and Chen Y: Antibacterial action of dispersed single-walled carbon nanotubes on Escherichia coli and Bacillus subtilis investigated by atomic force microscopy. Nanoscale 2: 2744-2750, 2010.

17. Ebbesen TW, Lezec HJ, Hiura H, Bennett JW, Ghaemi HF and Thio T: Electrical conductivity of individual carbon nanotubes. Nature 382: 54-56, 1996.

18. Treacy MMJ, Ebbesen TW and Gibson JM. Exceptionally high young's modulus observed for individual carbonnanotubes. Nature, 381: 678-680, 1996.

19. Iijima S: Helical microtubules of graphitic carbon. Nature 354: $56-58,1991$

20. Sharma S, Mehra NK, Jain K and Jain NK: Effect of functionalization on drug delivery potential of carbon nanotubes. Artif Cells Nanomed Biotechnol 44: 1851-1860, 2016.

21. Zhang B, Wang H, Shen S, She X, Shi W, Chen J, Zhang Q, Hu Y, Pang Z and Jiang X: Fibrin-targeting peptide CREKA-conjugated multi-walled carbon nanotubes for self-amplified photothermal therapy of tumor. Biomaterials 79: 46-55, 2016.

22. Bandyopadhyay A, Sarkar MD and Bhowmic AK: Poly(vinyl alcohol)/silica hybrid nanocomposites by sol-gel technique: Synthesis and properties. J Mater Sci 40: 5233-5241, 2005.

23. Rangari VK, Mohammad GM, Jeelani S, Hundley A, Vig K, Singh SR and Pillai S: Synthesis of Ag/CNT hybrid nanoparticles and fabrication of their Nylon-6 polymer nanocomposite fibers for antimicrobial applications. Nanotechnology 21: 095102, 2010.

24. Zheng Y, Huang X, Wang Y, Xu H and Chen X: Performance and characterization of irradiated poly(vinyl alcohol)/polyvinyl pyrrolidone composite hydrogels used as cartilages replacement. J Appl Polym Sci 113: 736-741, 2009.

25. Ma Y, Zheng Y, Huang X, Xi T, Lin X, Han D and Song W: Mineralization behavior and interface properties of BG-PVA/bone composite implants in simulated body fluid. Biomed Mater 5: 25003, 2010

26. Millon LE, Guhados G and Wan W: Anisotropic polyvinyl alcohol-bacterial cellulose nanocomposite for biomedical applications. J Biomed Mater Res B Appl Biomater 86: 444-452, 2008

27. Tsai YC and Huang JD: Poly(vinyl alcohol)-assisted dispersion of multiwalled carbon nanotubes in aqueous solution for electroanalysis. Electrochem Commun 8: 956-960, 2006.

28. Agrawal P, Soni S, Mittal G and Bhatnagar A: Role of polymeric biomaterials as wound healing agents. Int J Low Extrem Wounds 13: 180-190, 2014.

29. Abrigo M, McArthur SL and Kingshott P: Electrospun nanofibers as dressings for chronic wound care: Advances, challenges, and future prospects. Macromol Biosci 14: 772-792, 2014.

30. Ehrenreich M and Ruszczak Z: Tissue-engineered temporary wound coverings. Important options for the clinician. Acta Dermatovenerol Alp Panonica Adriat 15: 5-13, 2006.

31. Choi JS, Leong KW and Yoo HS: In vivo wound healing of diabetic ulcers using electrospun nanofibers immobilized with human epidermal growth factor (EGF). Biomaterials 29: 587-596, 2008.

32. Kim HS and Yoo HS: In vitro and in vivo EGF gene therapy for diabetic ulcers with electrospun fibrous meshes. Acta Biomater 9: 7371-7380, 2013

33. Kondo S and Kuroyanagi Y: Development of a wound dressing composed of hyaluronic acid and collagen sponge with epidermal growth factor. J Biomater Sci Polym Ed 23: 629-643, 2012. 